\title{
ZERO-FREE PRIMITIVE SQUARE REPRESENTATIONS BY SOME TERNARY QUADRATIC FORMS
}

\section{WERNER HÜRLIMANN}

Swiss Mathematical Society

University of Fribourg

CH-1700 Fribourg

Switzerland

e-mail: whurlimann@bluewin.ch

\begin{abstract}
The number of distinct zero-free primitive square representations by the ternary quadratic forms $x^{2}+b y^{2}+c z^{2}, b, c \in\{1,2,4,8\}$, is expressed in terms of the number of primitive square representations by this form taking into account zeros, permutations and sign changes, and the number of distinct primitive square representations by certain binary quadratic forms. Asymptotic expressions for the cumulative numbers are also derived and the obtained formulas are illustrated at two typical cases.
\end{abstract}

\section{Introduction}

A classical arithmetic problem concerns the representations of a number $m$ as a sum of $k \geq 2$ squares such that $x_{1}^{2}+x_{2}^{2}+\cdots+x_{k}^{2}=m$. The number of such representations, counting zeros, permutations and

2010 Mathematics Subject Classification: 11E25, 11D45.

Keywords and phrases: sum of squares primitive representation, ternary quadratic form, twisted Euler function, Bell ternary quadratic form, Cooper and Lam's conjecture.

Received March 22, 2016

(C) 2016 Scientific Advances Publishers 
sign changes, is denoted by $r_{k}(m)$. Similarly, the abbreviation $R_{k}(m)$ denotes the number of primitive representations of $m$ as a sum of $k \geq 2$ squares counting zeros, permutations and sign changes. The number of distinct primitive representations of $m$ as a sum of $k \geq 2$ non-zero squares such that $x_{1}^{2}+x_{2}^{2}+\cdots+x_{k}^{2}=m$ with $\prod_{j=1}^{k} x_{j} \neq 0$, which is denoted by $R_{k}^{d}(m)$, has been less studied. It is only recently that Hürlimann $[11,12,15]$ has derived formulas for $R_{k}^{d}\left(t^{2}\right), k=3,4,5$, the number of primitive Pythagorean quadruples, quintuples, and sextuples, respectively.

This previous work can be extended in another direction. Consider the number of integer solutions of the Diophantine equation $Q\left(x_{1}, x_{2}\right.$, $\left.\ldots, x_{k}\right)=m$ for given $m$ and arbitrary quadratic form $Q\left(x_{1}, x_{2}, \ldots, x_{k}\right)$. If one denotes by $r_{Q}(m)$ the total number of solutions, and by $R_{Q}(m)$ the total number of primitive solutions, one has the formula (e.g., Grosswald [9], Theorem 1, Section 1.1)

$$
r_{Q}(m)=\sum_{d^{2} \mid m} R_{Q}\left(\frac{m}{d^{2}}\right)
$$

Möbius inversion of (1.1) yields the formula (Cooper and Hirschhorn [5], Equation (1.3))

$$
\begin{aligned}
R_{Q}(m)= & r_{Q}(m)-\sum_{p_{1}^{2} \mid m} r_{Q}\left(\frac{m}{p_{1}^{2}}\right)+\sum_{p_{1}^{2}, p_{2}^{2} \mid m} r_{Q}\left(\frac{m}{p_{1}^{2} p_{2}^{2}}\right) \\
& -\sum_{p_{1}^{2}, p_{2}^{2}, p_{3}^{2} \mid m} r_{Q}\left(\frac{m}{p_{1}^{2} p_{2}^{2} p_{3}^{2}}\right)+\cdots+(-1)^{n} \sum_{p_{1}^{2}, \ldots, p_{n}^{2} \mid m} r_{Q}\left(\frac{m}{p_{1}^{2} \ldots p_{n}^{2}}\right),
\end{aligned}
$$

where $p_{1}, p_{2}, \ldots, p_{n}$ are the distinct primes whose squares divide $m$. 
In general, we are interested in the number $R_{Q}^{d}(m)$ of distinct primitive representations of $m$ by the quadratic form $Q\left(x_{1}, x_{2}, \ldots, x_{k}\right)$ without zeros such that $\prod_{j=1}^{k} x_{j} \neq 0$. Clearly, this number depends upon $R_{Q}(m)$, which is called principal component of $R_{Q}^{d}(m)$. For combinatorial reasons, a formula for $R_{Q}^{d}(m)$ will also depend upon other auxiliary components $R_{Q^{\prime}}^{d}(m)$ for various other quadratic forms $Q^{\prime}$ or degree less than $k$. For example, the number of primitive quadruples $R_{3}^{d}\left(t^{2}\right)$ depends besides the principal ternary component $R_{3}\left(t^{2}\right)$ on the auxiliary binary components $R_{Q_{1}}^{d}\left(t^{2}\right)$ and $R_{Q_{2}}^{d}\left(t^{2}\right)$ with $Q_{1}\left(x_{1}, x_{2}\right)=x_{1}^{2}+x_{2}^{2}$ and $\ell_{2}\left(x_{1}, x_{2}\right)=x_{1}^{2}+2 x_{2}^{2}$, respectively (see Hürlimann [11], Lemma 4). In the present work, we study the number $R_{Q}^{d}\left(t^{2}\right)$ for the ternary diagonal quadratic forms $Q(x, y, z)=x^{2}+b y^{2}+c z^{2} \quad$ with $\quad b, c \in\{1,2,4,8\}$, $b \leq c,(b, c) \neq(1,1)$. Instead of $r_{Q}(m), R_{Q}(m)$, and $R_{Q}^{d}(m)$ we use the notations $r_{(1, b, c)}(m), R_{(1, b, c)}(m)$, and $R_{(1, b, c)}^{d}(m)$. Similarly, for the binary quadratic forms $Q(x, y)=a x^{2}+b y^{2},(a, b) \neq(1,1)$, we write $r_{(a, b)}(m), R_{(a, b)}(m)$, and $R_{(a, b)}^{d}(m)$ for $r_{Q}(m), R_{Q}(m)$, and $R_{Q}^{d}(m)$, respectively.

Recall that $r_{(1, b, c)}(m)$ has been first determined by Bell [2] in terms of the three-squares counting formula $r_{3}(m)$ only. A modern proof of this result is presented in Hürlimann [13]. Now, by Möbius inversion (1.2), the principal component $R_{(1, b, c)}\left(t^{2}\right)$ depends upon the knowledge of $r_{(1, b, c)}\left(t^{2}\right)$, whose value is predicted by the conjecture of Cooper and Lam 
[6]. A complete and elementary proof of this conjecture for Bell's ternary quadratic forms has been given in Hürlimann [14]. Guo et al. [10] and Ye [24] obtained further partial results, and seemingly Kim and Oh [20] resolved this conjecture completely. It turns out that $r_{(1, b, c)}\left(t^{2}\right)$, is determined by constant multiples of $r_{3}\left(n^{2}\right)$ or $r_{3}\left(2 n^{2}\right)$. A formula for $r_{3}\left(n^{2}\right)$ is due to Hurwitz [16]:

$$
r_{3}\left(n^{2}\right)=6 \cdot S(m), \text { with } S(m)=\prod_{p \mid m}\left\{\sigma\left(p^{\lambda p}\right)-\left(\frac{-1}{p}\right) \sigma\left(p^{\lambda p^{-1}}\right)\right\},
$$

where $\sigma(m)$ counts the sum of all positive divisors of $m,\left(\frac{-1}{p}\right)$ is the Legendre symbol, and $n=2^{\lambda_{2}} \cdot m, m=\prod_{p \mid m} p^{\lambda_{p}}, \quad$ is the unique decomposition of $n$ in prime numbers $p$ (see Dickson [7], p. 271, and Hürlimann [14], Remarks 2.1). Similarly, in the same notations, one has the formula (see Hürlimann [14], Lemma 2.1)

$$
r_{3}\left(2 n^{2}\right)=12 \cdot T(m), \text { with } T(m)=\prod_{p \mid m}\left\{\sigma\left(p^{\lambda p}\right)-\left(\frac{-2}{p}\right) \sigma\left(p^{\lambda_{p}-1}\right)\right\} .
$$

To determine the auxiliary components of the counting function $R_{(1, b, c)}^{d}\left(t^{2}\right)$, we apply the method already used in previous papers by the author. A brief description of the content follows.

Section 2 derives the exact formulas for the counting functions $R_{(1, b, c)}^{d}(m)$ as additive functions of the principal ternary and auxiliary binary quadratic components. In Section 4, we recall the expressions for the counting function $r_{(1, b, c)}\left(t^{2}\right)$ predicted by Cooper and Lam's conjecture, whose proof for Bell's ternary quadratic forms has been completed in Hürlimann [14]. Using Möbius inversion, we obtain in 
Theorem 3.2 expressions for the principal component $R_{(1, b, c)}\left(t^{2}\right)$ that depend on two twisted Euler (totient) functions with Dirichlet characters of period four and eight, respectively. Asymptotic expressions for the partial sums $\sum R_{(1, b, c)}\left(t^{2}\right)$ over all positive odd integers, which are required to approximate the cumulative number of all primitive square representations without zeros by the forms $x^{2}+b y^{2}+c z^{2}$, are displayed in the formulas (3.8) and (3.10). Section 4 derives formulas that enable the calculation of all 14 auxiliary binary quadratic components. Finally, Section 5 illustrates numerically the obtained exact and asymptotic counting formulas at the two ternary quadratic forms $x^{2}+y^{2}+2 z^{2}$ and $x^{2}+2 y^{2}+2 z^{2}$.

\section{Algebraic Combinatorial Derivation of the Counting Formulas}

The basic method is introduced as follows. Consider the $R_{(1, b, c)}(m)$ integer triples $(x, y, z)$ solving $x^{2}+b y^{2}+c z^{2}=m, 1 \leq b \leq c$, taking into account zeros, permutations and sign changes. The solutions are said to be primitive if the condition $\operatorname{gcd}(x, y, z, m)=1$ is satisfied. Note the convention (made throughout) that any integer is a divisor of zero. Looking at possible zeros and equal entries the distinct primitive solutions can take 8 (partially) different forms, namely, $(x, y, z),(x, y, y),(x, x, z),(z, z, x),(x, y, x),(x, y, 0),(x, 0, z)$, and $(0, y, z)$, with distinct entries $x, y, z \neq 0$. For each form, one must determine the number of resulting representations counting permutations and sign changes, as well as the number of distinct primitive solutions generated by this form. The latter counting function is denoted by $D_{\bullet}(m)$, where stands for the quadratic form type of the corresponding Diophantine equation. By convention, we set $D_{2}(m)=D_{(1,1)}(m)$ and $D_{3}(m)=D_{(1,1,1)}(m)$. 
Depending on the values of $1 \leq b \leq c$, these forms may allow for permutations. The middle forms $(x, x, z),(z, z, x)$, and $(x, y, x)$, are not allowed to be permuted. The use of $(z, z, x)$ or $(x, y, x)$ as different from $(x, x, z)$ is justified when the forms $(x, x, z)$ and $(z, z, x)$, respectively $(x, x, z)$ and $(x, y, x)$, generate distinct solutions albeit they solve the same Diophantine equation. This occurs for the special values $(b, c)=(1,2)$ for the use of $(z, z, x)$, respectively $1<b \leq c$ for the use of $(x, y, x)$. The required information is summarized in Table 2.1. For convenience, if nothing else is mentioned, we omit the argument $m$ in the different counting functions. 
Table 2.1. Forms, Diophantine equations, representations and distinct solutions for $R_{(1, b, c)}$

\begin{tabular}{|c|c|c|c|c|c|c|}
\hline Form & Conditions on $b, c$ & Diophantine Eq. & $\begin{array}{c}\# \\
\text { perm. }\end{array}$ & $\begin{array}{c}\# \\
\text { sign } \\
\text { changes }\end{array}$ & $\begin{array}{c}\text { total \# } \\
\text { representations }\end{array}$ & $\begin{array}{c}\# \\
\text { distinct } \\
\text { solutions }\end{array}$ \\
\hline$(x, y, z)$ & $b=c=1$ & $x^{2}+b y^{2}+c z^{2}=m$ & 6 & 8 & 48 & $D_{(1, b, c)}$ \\
& $1=b<c, 1<b=c$ & & 2 & 8 & 16 & \\
\hline$(x, y, y)$ & $b=c=1$ & $x^{2}+(b+c) y^{2}=m$ & 3 & 8 & 24 & $D_{(1, b+c)}$ \\
& $b=1<c$ & & 2 & 8 & 16 & \\
\hline$(x, x, z)$ & $(b, c) \neq(1,1)$ & $(1+b) x^{2}+c z^{2}=m$ & 1 & 8 & 8 & $D_{(1+b, c)}$ \\
\hline$(z, z, x)$ & $(b, c)=(1,2)$ & $2 z^{2}+2 x^{2}=m$ & 1 & 8 & 8 & $D_{(2,2)}$ \\
\hline$(x, y, x)$ & $1<b \leq c$ & $(1+b) x^{2}+c z^{2}=m$ & 1 & 8 & 8 & $D_{(b, 1+c)}$ \\
\hline$(x, y, 0)$ & $b=c=1$ & $x^{2}+b y^{2}=m$ & 6 & 4 & 24 & $D_{(1, b)}$ \\
& $1=b<c$ & & 2 & 4 & 8 & \\
& $1<b \leq c$ & & 1 & 4 & 4 & \\
\hline$(x, 0, z)$ & $1<b<c$ & $x^{2}+c z^{2}=m$ & 1 & 4 & 4 & $D_{(1, c)}$ \\
\hline$(0, y, z)$ & $1=b<c, 1<b=c$ & $b y^{2}+c z^{2}=m$ & 2 & 4 & 8 & $D_{(b, c)}$ \\
& $1<b<c$ & & 1 & 4 & 4 & \\
\hline
\end{tabular}


Due to the conditions on $b, c$ five cases must be considered. First, by definition of $R_{(1, b, c)}^{d}$, one obtains from the first five forms without zeros in Table 2.1 the identities

$$
\begin{aligned}
& D_{3}+D_{(1,2)}=R_{3}^{d}, \quad(b, c)=(1,1), \\
& D_{(1,1,2)}+D_{(1,3)}+2 D_{(2,2)}=R_{(1,1,2)}^{d}, \quad(b, c)=(1,2), \\
& D_{(1,1, c)}+D_{(1,1+c)}+D_{(2, c)}=R_{(1,1, c)}^{d}, \quad 1=b, 2<c, \\
& D_{(1, b, b)}+D_{(1,2 b)}+2 D_{(b, 1+b)}=R_{(1, b, b)}^{d}, \quad 1<b=c, \\
& D_{(1, b, c)}+D_{(1, b+c)}+D_{(1+b, c)}+D_{(b, 1+c)}=R_{(1, b, c)}^{d}, \quad 1<b<c .
\end{aligned}
$$

On the other hand, by definition of $R_{(1, b, c)}$ and based on Table 2.1, one has the identities

$$
\begin{aligned}
& 48 D_{3}+24 D_{(1,2)}+24 D_{2}=R_{3}, \quad(b, c)=(1,1), \\
& 16 D_{(1,1,2)}+16 D_{(1,3)}+16 D_{(2,2)}+8 D_{2}+8 D_{(1,2)}=R_{(1,1,2)}, \quad(b, c)=(1,2) \\
& 16 D_{(1,1, c)}+16 D_{(1,1+c)}+8 D_{(2, c)}+8 D_{2}+8 D_{(1, c)}=R_{(1,1, c)}, \quad 1=b, 2<c \\
& 16 D_{(1, b, b)}+8 D_{(1,2 b)}+16 D_{(b, 1+b)}+8 D_{(1, b)}+8 D_{(b, b)}=R_{(1, b, b)}, \quad 1<b=c, \\
& 8 D_{(1, b, c)}+8 D_{(1, b+c)}+8 D_{(1+b, c)}+8 D_{(b, 1+c)}+4 D_{(1, b)}+4 D_{(1, c)}+4 D_{(b, c)} \\
& =R_{(1, b, c)}, \quad 1<b<c .
\end{aligned}
$$

Solving (2.1)-(2.5) for $D_{(1, b, c)}$ and inserting into (2.6)-(2.10) one gets after straightforward calculation the desired expressions for $R_{(1, b, c)}^{d}$. 
Theorem 2.1. The number of non-trivial distinct primitive representations of a number by the ternary quadratic forms $x^{2}+b y^{2}+c z^{2}$, $1 \leq b \leq c$, satisfies the following parametric formulas:

$$
\begin{aligned}
& 48 R_{3}^{d}=R_{3}+24 D_{(1,2)}-24 D_{2}, \quad(b, c)=(1,1), \\
& 16 R_{(1,1,2)}^{d}=R_{(1,1,2)}+16 D_{(2,2)}-8 D_{2}-8 D_{(1,2)}, \quad(b, c)=(1,2), \\
& 16 R_{(1,1, c)}^{d}=R_{(1,1, c)}+8 D_{(2, c)}-8 D_{2}-8 D_{(1, c)}, \quad 1=b, 2<c, \\
& 16 R_{(1, b, b)}^{d}=R_{(1, b, b)}+8 D_{(1,2 b)}+16 D_{(b, 1+b)}-8 D_{(1, b)}-8 D_{(b, b)}, \\
& 1<b=c, \\
& 8 R_{(1, b, c)}^{d}=R_{(1, b, c)}-4 D_{(1, b)}-4 D_{(1, c)}-4 D_{(b, c)}, \quad 1<b<c .
\end{aligned}
$$

\section{Principal Ternary Quadratic Components}

To obtain formulas for the principal components $R_{(1, b, c)}\left(t^{2}\right)$, one applies the Möbius inversion formula (1.2) to the counting function $r_{(1, b, c)}\left(t^{2}\right)$, whose expression follows from the validity of Cooper and Lam's conjecture for Bell's ternary quadratic forms.

Theorem 3.1. Let $n=2^{\lambda_{2}} \cdot m$ with $m$ odd. The counting function $r_{(1, b, c)}\left(n^{2}\right)$ for Bell's ternary quadratic forms is determined by the Table 3.1 below. 
Table 3.1. The counting function $r_{(1, b, c)}\left(n^{2}\right)$ in dependence of $n(\bmod 4)$ and $S(m), T(m)$

\begin{tabular}{|l|l|l|l|l|l|l|l|l|l|}
\hline \multirow{2}{*}{$\begin{array}{l}\text { class } n \\
\bmod 4\end{array}$} & \multicolumn{7}{|c|}{ Parameters $b, c \in\{1,2,4,8\}$} \\
\cline { 2 - 10 } & $(1,2)$ & $(1,4)$ & $(1,8)$ & $(2,2)$ & $(2,4)$ & $(2,8)$ & $(4,4)$ & $(4,8)$ & $(8,8)$ \\
\hline 1,3 & $4 T(m)$ & $4 S(m)$ & $4 T(m)$ & $2 S(m)$ & $2 T(m)$ & $2 S(m)$ & $2 S(m)$ & $2 T(m)$ & $2 S(m)$ \\
\hline 2 & $12 T(m)$ & $6 S(m)$ & $4 T(m)$ & $6 S(m)$ & $4 T(m)$ & $2 S(m)$ & $6 S(m)$ & $4 T(m)$ & $2 S(m)$ \\
\hline 0 & $12 T(m)$ & $6 S(m)$ & $12 T(m)$ & $6 S(m)$ & $12 T(m)$ & $6 S(m)$ & $6 S(m)$ & $12 T(m)$ & $6 S(m)$ \\
\hline
\end{tabular}

Proof. This is shown in Hürlimann [14], Theorem 2.2.

The description of the principal components can be done using two twisted Euler (totient) functions with Dirichlet characters of period four and eight, respectively. Let $t>2$ be an odd number, and let $p$ denote a prime number. Then, one defines

$$
\varphi_{4}(t)=t \cdot \prod_{p \mid t}\left(1-\chi_{4}(p) / p\right), \text { with } \chi_{4}(p)= \begin{cases}0, & p=2, \\ 1, & p \equiv 1(\bmod 4), \\ -1, & p \equiv 3(\bmod 4),\end{cases}
$$

and

$$
\varphi_{8}(t)=t \cdot \prod_{p \mid t}\left(1-\chi_{8}(p) / p\right), \text { with } \chi_{8}(p)=\left\{\begin{array}{l}
1, \text { if } p \equiv 1 \text { or } 3(\bmod 8) \\
-1, \text { if } p \equiv 5 \text { or } 7(\bmod 8) .
\end{array}\right.
$$

Theorem 3.2. The counting function $R_{(1, b, c)}\left(t^{2}\right), t=2^{\lambda_{2}} s>2$, sodd, for Bell's ternary quadratic forms is determined as follows. If $\lambda_{2} \geq 3$, then one has $R_{(1, b, c)}\left(t^{2}\right)=0$. Otherwise, the counting function takes the values listed in the Table 3.2 below. 
Table 3.2. The counting function $R_{(1, b, c)}\left(t^{2}\right), t>2$, in dependence of $t$ and $\varphi_{4}(t), \varphi_{8}(t)$

\begin{tabular}{|l|c|c|c|c|c|c|c|c|c|}
\hline \multirow{2}{*}{$\begin{array}{l}\text { Value } \\
\text { of } t\end{array}$} & \multicolumn{10}{|c|}{ Parameters $b, c \in\{1,2,4,8\}$} \\
\cline { 2 - 10 } & $(1,2)$ & $(1,4)$ & $(1,8)$ & $(2,2)$ & $(2,4)$ & $(2,8)$ & $(4,4)$ & $(4,8)$ & $(8,8)$ \\
\hline$t=s$ & $4 \varphi_{8}(t)$ & $4 \varphi_{4}(t)$ & $4 \varphi_{8}(t)$ & $2 \varphi_{4}(t)$ & $2 \varphi_{8}(t)$ & $2 \varphi_{4}(t)$ & $2 \varphi_{4}(t)$ & $2 \varphi_{8}(t)$ & $2 \varphi_{4}(t)$ \\
\hline$t=2 s$ & $8 \varphi_{8}(s)$ & $2 \varphi_{4}(s)$ & 0 & $4 \varphi_{4}(s)$ & $2 \varphi_{8}(s)$ & 0 & $4 \varphi_{4}(s)$ & $2 \varphi_{8}(s)$ & 0 \\
\hline$t=4 s$ & 0 & 0 & $8 \varphi_{8}(s)$ & 0 & $8 \varphi_{8}(s)$ & $4 \varphi_{4}(s)$ & 0 & $8 \varphi_{8}(s)$ & $4 \varphi_{4}(s)$ \\
\hline
\end{tabular}


Proof. This is obtained from Theorem 3.1 through routine application of Möbius inversion and is therefore omitted.

In applications, the exact calculation of the twisted Euler functions requires a factorization table for all odd numbers into distinct prime factors. Since factorization is a complex algorithm, one is also interested in asymptotic formulas. It turns out to be more efficient to study the partial sums below $x$ of the twisted Euler functions over all positive natural numbers $2 \leq n \leq x$ (including even numbers), which is denoted by

$$
\Phi_{k}(x)=\sum_{2 \leq n \leq x} \varphi_{k}(n), \quad k=4,8 .
$$

These kinds of sums have been recently studied by Kaczorowski [17] and Kaczorowski and Wiertelak [18, 19]. In our context, one must additionally account for a separation between sums over odd and even numbers. Consider the partial sums of the twisted Euler functions over odd and even numbers denoted by

$$
\Phi_{k}^{0}(x)=\sum_{3 \leq \text { odd }} \varphi_{k \leq x}(n), \Phi_{k}^{e}(x)=\sum_{2 \leq \text { even }} \varphi_{n \leq x}(n), \text { with } \Phi_{k}(x)=\Phi_{k}^{0}(x)+\Phi_{k}^{e}(x) .
$$

Lemma 3.1. The odd partial sums in (3.4) are determined by the formula $\Phi_{k}^{0}(x)=\Phi_{k}(x)-2 \cdot\left\{1+\Phi_{k}\left(\frac{1}{2} x\right)\right\}$.

Proof. This follows from Lemma 5 in Hürlimann [11].

On the other hand, for fixed $k=4,8$, the twisted Euler function $\varphi_{k}(n)$ is related to a Dirichlet L-function via its Euler product through the identity

$$
L\left(s, \chi_{k}\right)=\sum_{n=1}^{\infty} \frac{\chi_{k}(n)}{n^{s}}=\prod_{p}\left(1-\chi_{k}(p) / p^{s}\right)^{-1}, \quad \operatorname{Re}(s)>1,
$$

and the following result about sums of twisted Euler functions. 
Theorem 3.3. For all $x \geq 1$, one has the asymptotic relationship

$$
\widetilde{\Phi}_{k}(x)=\sum_{1 \leq n \leq x} \varphi_{k}(n)=\frac{1}{2} L\left(2, \chi_{k}\right)^{-1} \cdot x^{2}+O(x \ln (2 x)) .
$$

Proof. See Kaczorowski and Wiertelak [18], and Kaczorowski [17], Theorem 1.1.

In our situation, this applies to the Dirichlet characters in (3.1)-(3.2). For the period four, one has the asymptotic formula $\Phi_{4}(x) \sim \widetilde{\Phi}_{4}(x) \sim$ $(2 G)^{-1} \cdot x^{2}(x \rightarrow \infty)$, with Catalan's constant

$$
G=L\left(2, \chi_{4}\right)=\sum_{n=0}^{\infty} \frac{(-1)^{n}}{(2 n+1)^{2}} \doteq 0.915965594177
$$

With Lemma 3.1, one obtains further the asymptotic relationship (see also Hürlimann [11], Section 3)

$$
\Phi_{4}^{0}(x) \sim(4 G)^{-1} \cdot x^{2} \quad(x \rightarrow \infty) .
$$

For the Dirichlet character of period eight, one has $\Phi_{8}(x) \sim \widetilde{\Phi}_{8}(x) \sim$ $(2 H)^{-1} \cdot x^{2}(x \rightarrow \infty)$ with

$$
\begin{aligned}
H & =L\left(2, \chi_{8}\right)=\frac{1}{64}\left\{\psi_{1}\left(\frac{1}{8}\right)+\psi_{1}\left(\frac{3}{8}\right)-\psi_{1}\left(\frac{5}{8}\right)-\psi_{1}\left(\frac{7}{8}\right)\right\} \\
& \doteq \frac{1}{64}\{65.388132+8.161776-3.401182-2.00574\} \doteq 1.064734,
\end{aligned}
$$

where $\psi_{1}(z)=\psi^{\prime}(z)=\frac{d^{2}}{d z^{2}} \ln \Gamma(z)=\sum_{n=0}^{\infty}(z+n)^{-2}=\zeta(2, z)$ is the trigamma function, which is known to coincide with the Hurwitz zeta function (e.g., Weisstein [23], Equation (42)). Again, with Lemma 3.1, one obtains

$$
\Phi_{8}^{0}(x) \sim(4 H)^{-1} \cdot x^{2} \quad(x \rightarrow \infty) .
$$


Remarks 3.1. According to Bailey et al. [1], Catalan's constant is "the most basic constant whose irrationality and transcendence (through strongly suspected) remain unproven". Besides the information provided in Hürlimann [11] there is also a formula due to Ramanujan and quite recent formulas in the BPP compendium lists used to compute it. Bailey et al. [1] believe that their Equation (18) is the most economical formula known for computing Catalan's constant. Concerning the constant (3.9), we note that values of the polygamma functions at rational arguments have been extensively studied by Choi and Cvijovic [3, 4].

\section{Auxiliary Binary Quadratic Components}

The formulas (2.9)-(2.12) show that the number of distinct primitive square representations by Bell's ternary quadratic forms without zeros depends upon the following 14 binary quadratic components:

$$
\begin{aligned}
D_{2}= & D_{(1,1)}, D_{(1,2)}, D_{(1,4)}, D_{(1,8)}, D_{(1,16)}, D_{(2,2)}, D_{(2,3)}, \\
& D_{(2,4)}, D_{(2,8)}, D_{(4,4)}, D_{(4,5)}, D_{(4,8)}, D_{(8,8)}, D_{(8,9)} .
\end{aligned}
$$

In general, to determine the number of distinct primitive solutions of the equations $b x^{2}+c y^{2}=t^{2}$, one first finds a formula for the number of all representations $r_{(b, c)}\left(t^{2}\right)$ taking into account zeros, permutations and sign changes, and then apply Möbius inversion to get an expression for

$R_{(b, c)}\left(t^{2}\right)$, from which one gets $D_{(b, c)}\left(t^{2}\right)=\frac{1}{4} R_{(b, c)}\left(t^{2}\right)$ in case $c \neq b$ or $D_{(b, b)}\left(t^{2}\right)=\frac{1}{8} R_{(b, b)}\left(t^{2}\right)$ in case $c=b$. We suppose that the positive integer $t \geq 2$ has the prime factorization $t=2^{\lambda_{2}} \cdot \prod_{i=1}^{m} p_{i}^{\lambda_{i}}$.

Some counting functions have been determined in our previous work (Hürlimann [12], Equation (4.1)-(4.3), Hürlimann [15], Section 3). One has 


$$
\begin{aligned}
& D_{2}\left(t^{2}\right)=\left\{\begin{array}{l}
2^{m-1}, \text { if } p_{i} \equiv 1(\bmod 4), i=1, \ldots, m, \lambda_{2}=0 \\
0, \quad \text { otherwise }
\end{array}\right. \\
& D_{(1,2)}\left(t^{2}\right)= \begin{cases}2^{m-1}, & \text { if } p_{i} \equiv 1,3(\bmod 8), i=1, \ldots, m, \lambda_{2}=0, \\
0, & \text { otherwise }\end{cases} \\
& D_{(2,2)}\left(t^{2}\right)=\left\{\begin{array}{l}
D_{2}\left(s^{2}\right), \text { if } t=2 s, s \text { odd }, \\
0, \quad \text { otherwise }
\end{array}\right. \\
& D_{(2,3)}\left(t^{2}\right)=0, D_{(1,4)}\left(t^{2}\right)=\left\{\begin{array}{l}
D_{2}\left(t^{2}\right), \quad \text { if } t \text { odd }, \\
D_{2}\left(s^{2}\right), \text { if } t=2 s, s \text { odd }, \\
0, \quad \text { otherwise. }
\end{array}\right.
\end{aligned}
$$

Furthermore, one shows without difficulty the following reduction formulas:

$$
\begin{aligned}
& D_{(4,4)}\left(t^{2}\right)=D_{(2,2)}\left(t^{2}\right)= \begin{cases}D_{2}\left(s^{2}\right), & \text { if } t=2 s, s \text { odd }, \\
0, & \text { otherwise, }\end{cases} \\
& D_{(4,8)}\left(t^{2}\right)=D_{(2,4)}\left(t^{2}\right)= \begin{cases}D_{(1,2)}\left(s^{2}\right), \text { if } t=2 s, s \text { odd }, \\
0, & \text { otherwise, }\end{cases} \\
& D_{(8,8)}\left(t^{2}\right)=D_{(2,8)}\left(t^{2}\right)= \begin{cases}D_{2}\left(s^{2}\right), \text { if } t=4 s, s \text { odd }, \\
0, & \text { otherwise. }\end{cases}
\end{aligned}
$$

Next, we derive a reduction formula for $D_{(8,9)}$. If $t$ is even, then one has

$$
D_{(8,9)}\left(t^{2}\right)=D_{(2,9)}\left((t / 2)^{2}\right)=\left\{\begin{array}{l}
D_{(1,2)}\left((t / 6)^{2}\right), \quad \text { if } 6 \mid t \\
0, \quad \text { otherwise }
\end{array}\right.
$$


where $D_{(8,9)}\left(t^{2}\right)=0$ follows because $t^{2} \equiv 8(\bmod 9)$ is impossible when $t$ is not divisible by 3 . Similarly, if $t$ is odd, then one obtains

$$
D_{(8,9)}\left(t^{2}\right)= \begin{cases}D_{(1,8)}\left((t / 3)^{2}\right), & \text { if } 3 \mid t, \\ 0, & \text { otherwise }\end{cases}
$$

Together, this shows the counting formula

$$
D_{(8,9)}\left(t^{2}\right)= \begin{cases}D_{(1,8)}\left((t / 3)^{2}\right), & \text { if } t \text { odd }, 3 \mid t \\ D_{(1,2)}\left((t / 6)^{2}\right), & \text { if } 6 \mid t \\ 0, & \text { otherwise. }\end{cases}
$$

Counting formulas for the remaining three binary quadratic forms are determined using some general results of Sun and Williams [22] for the class number 2 . We begin with $D_{(1,8)}$. If $t$ is even one has $D_{(1,8)}\left(t^{2}\right)$ $=D_{(1,2)}\left((t / 2)^{2}\right)$. Let now $t$ be odd. The binary quadratic form $x^{2}+8 y^{2}$ of discriminant $d=-32$, conductor $f=2$, has class number 2 , and satisfies the counting formula (use Sun and Williams [22], Theorem 9.3, (iii))

$$
r_{(1,8)}\left(t^{2}\right)=2 \cdot \prod_{p_{i} \equiv 1,3(\bmod 8)}\left(1+2 \lambda_{i}\right) .
$$

Through Möbius inversion one sees that $R_{(1,8)}\left(t^{2}\right)=4 \cdot 2^{m-1}$, if $p_{i} \equiv 1$, $3(\bmod 8), i=1, \ldots, m$, and $R_{(1,8)}\left(t^{2}\right)=0$, otherwise. Comparing with (4.3), one obtains the reduction formula

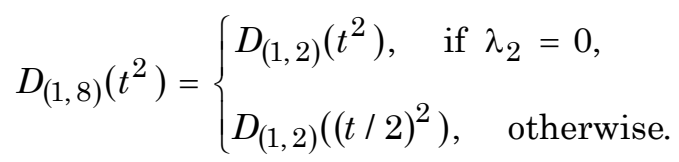


In the same manner, one shows the reduction formula

$$
D_{(1,16)}\left(t^{2}\right)=\left\{\begin{array}{l}
D_{2}\left(t^{2}\right), \quad \text { if } \lambda_{2}=0 \\
D_{(1,4)}\left((t / 2)^{2}\right), \quad \text { otherwise }
\end{array}\right.
$$

Indeed, if $t$ is even the result is immediate. In case $t$ is odd, the form $x^{2}+16 y^{2}$ of discriminant $d=-64$, conductor $f=4$, has class number 2 , and satisfies the counting formula (use Sun and Williams [22], Theorem 9.3, (iii))

$$
r_{(1,8)}\left(t^{2}\right)=2 \cdot \prod_{p_{i} \equiv 1(\bmod 4)}\left(1+2 \lambda_{i}\right) .
$$

Again, applying Möbius inversion one gets $R_{(1,16)}\left(t^{2}\right)=4 \cdot 2^{m-1}$, if $p_{i} \equiv 1(\bmod 4), i=1, \ldots, m$, and $R_{(1,8)}\left(t^{2}\right)=0$, otherwise. Comparing with (4.2), the formula (4.11) follows.

Finally, consider the binary quadratic form $4 x^{2}+5 y^{2}$. If $t$ is even one has $D_{(4,5)}\left(t^{2}\right)=D_{(1,5)}\left((t / 2)^{2}\right)$, and if $t$ is odd one has $D_{(4,5)}\left(t^{2}\right)=$ $D_{(1,5)}\left(t^{2}\right)$. The latter follows by observing that the non-trivial solutions of $4 x^{2}+5 y^{2}=t^{2}$ are identical to those of $x^{2}+5 y^{2}=t^{2}$ because $X=2 x$ must be even in case $t$ is odd. Together, this shows the reduction formula

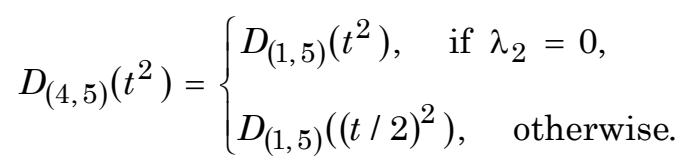

Now, the form $x^{2}+5 y^{2}$ of discriminant $d=-20$, conductor $f=2$, has class number 2, and satisfies for odd $t$ the counting formula (use Sun and Williams [22], Theorem 9.3, (iii), see also Dickson [8], Exercises XXIII, no. 1, p.84, Section 53) 


$$
r_{(1,5)}\left(t^{2}\right)=2 \cdot \prod_{\left(\frac{-5}{p_{i}}\right)=1}\left(1+2 \lambda_{i}\right) .
$$

Applying Möbius inversion and using the quadratic reciprocity law, one obtains the formula

$$
D_{(1,5)}\left(t^{2}\right)= \begin{cases}2^{m-1}, & \text { if } p_{i} \equiv 1,3,7,9(\bmod 20), i=1, \ldots, m, \lambda_{2}=0 \\ 0, & \text { otherwise }\end{cases}
$$

Together with (4.12), the formula (4.13) completely determines $D_{(4,5)}\left(t^{2}\right)$.

Finally, asymptotic formulas for partial sums of the type $N_{(b, c)}(x)=$ $\sum_{t \leq x} D_{(b, c)}\left(t^{2}\right)$ can be derived using an old method by Lehmer [21]. For example, one has

$$
N_{2}(x) \sim \frac{x}{2 \pi}, \quad N_{(1,2)}(x) \sim \frac{\sqrt{2} \cdot x}{2 \pi} \text { as } x \rightarrow \infty .
$$

\section{Numerical Examples}

Let us illustrate the obtained exact and asymptotic counting formulas at the two ternary quadratic forms $x^{2}+y^{2}+2 z^{2}$ and $x^{2}+2 y^{2}+2 z^{2}$, which generalize the numerical results obtained in Hürlimann [11], Table 1, for the form $x^{2}+y^{2}+z^{2}$. With Theorem 2.1, Table 3.2, and the formulas of Section 4, one obtains the formula (see also Hürlimann [12], Proposition 3.1) 


$$
R_{(1,1,2)}^{d}\left(t^{2}\right)=\left\{\begin{array}{l}
\frac{1}{4} \varphi\left(t, \chi_{8}\right)-\frac{1}{2} D_{2}\left(t^{2}\right)-\frac{1}{2} D_{(1,2)}\left(t^{2}\right), \quad \text { if } t>2 \text { is odd } \\
\frac{1}{2} \varphi\left(s, \chi_{8}\right)+D_{2}\left(s^{2}\right), \quad \text { if } t=2 s, s>1 \text { odd } \\
1, \quad \text { if } t=2, \\
0, \quad \text { if } 4 \mid t
\end{array}\right.
$$

Asymptotically, as $x \rightarrow \infty$, one must distinguish between partial sums over odd number and over numbers exactly divisible by two. Application of (3.10) and (4.14) yields the formulas

$$
\begin{aligned}
& N_{(1,1,2)}^{\text {odd }}(x)=\sum_{\substack{2<t \leq x \\
t \text { odd }}} R_{(1,1,2)}^{d}\left(t^{2}\right)=\frac{1}{4} \Phi_{8}^{0}(x)-\frac{1}{2}\left\{N_{(1,2)}(x)+N_{2}(x)\right\} \sim \frac{x^{2}}{16 H} \\
& -\frac{(\sqrt{2}+1) x}{4 x}, \\
& N_{(1,1,2)}^{2 \cdot \text { odd }}(x)=\sum_{\substack{2<2 \leq \leq x \\
s \text { odd }}} R_{(1,1,2)}^{d}\left(4 s^{2}\right)=\frac{1}{2} \Phi_{8}^{0}\left(\frac{x}{2}\right)+N_{2}\left(\frac{x}{2}\right) \sim \frac{x^{2}}{32 H}+\frac{x}{4 \pi}, \\
& N_{(1,1,2)}(x)=\sum_{2 \leq t \leq x} R_{(1,1,2)}^{d}\left(t^{2}\right) \sim \frac{3 x^{2}}{32 H}-\frac{(\sqrt{2} x)}{4 x} .
\end{aligned}
$$

Table 5.1 illustrates numerically. Similarly, one obtains (see also Hürlimann [15], Proposition 4.2)

$$
R_{(1,2,2)}^{d}\left(t^{2}\right)=\left\{\begin{array}{l}
\frac{1}{8} \varphi\left(t, \chi_{4}\right)+\frac{1}{2} D_{2}\left(t^{2}\right)-\frac{1}{2} D_{(1,2)}\left(t^{2}\right), \quad \text { if } t>2 \text { is odd } \\
\frac{1}{4} \varphi\left(s, \chi_{4}\right), \quad \text { if } t=2 s, s>1 \text { odd } \\
0, \quad \text { if } t=2 \text { or } 4 \mid t .
\end{array}\right.
$$


and the asymptotic formulas

$$
\begin{aligned}
& N_{(1,2,2)}^{\text {odd }}(x)=\sum_{\substack{2<t \leq x \\
t \text { odd }}} R_{(1,2,2)}^{d}\left(t^{2}\right)=\frac{1}{8} \Phi_{4}^{0}(x)-\frac{1}{2}\left\{N_{(1,2)}(x)-N_{2}(x)\right\} \sim \frac{x^{2}}{32 G} \\
& N_{(1,2,2)}^{2 \cdot \text { odd }}(x)=\sum_{\substack{2<2 s \leq x \\
s \text { odd }}} R_{(1,2,2)}^{d}\left(4 s^{2}\right)=\frac{1}{4} \Phi_{4}^{0}\left(\frac{x}{2}\right) \sim \frac{x^{2}}{64 G} \\
& N_{(1,2,2)}(x)=\sum_{2 \leq t \leq x} R_{(1,2,2)}^{d}\left(t^{2}\right) \sim \frac{3 x^{2}}{64 G}-\frac{(\sqrt{2}-1) x}{4 \pi} .
\end{aligned}
$$

Table 5.2 illustrates numerically. 
Table 5.1. Cumulative number of non-trivial primitive solutions of $x^{2}+y^{2}+2 z^{2}=t^{2}$

\begin{tabular}{|c|c|c|c|c|c|c|c|c|}
\hline limit $x$ & $\begin{array}{c}\text { exact } \\
\text { odd } \\
\text { count }\end{array}$ & $\begin{array}{c}\text { exact } \\
2 \times \text { odd } \\
\text { count }\end{array}$ & $\begin{array}{c}\text { exact } \\
\text { count }\end{array}$ & $\begin{array}{c}\text { asymptotic } \\
\text { odd count }\end{array}$ & $\begin{array}{c}\text { asymptotic } \\
2 \times \text { odd } \\
\text { count }\end{array}$ & $\begin{array}{c}\text { asymptotic } \\
\text { count }\end{array}$ & $\begin{array}{c}\text { signed } \\
\text { difference }\end{array}$ & $\begin{array}{c}\text { percentage } \\
\text { relative } \\
\text { error }\end{array}$ \\
\hline 100 & 563 & 307 & 870 & 568 & 301 & 869 & -1 & $0.11 \%$ \\
\hline 200 & 2315 & 1182 & 3497 & 2310 & 1190 & 3500 & 3 & $0.09 \%$ \\
\hline 300 & 5221 & 2664 & 7885 & 5225 & 2665 & 7890 & 5 & $0.06 \%$ \\
\hline 400 & 9307 & 4739 & 14046 & 9315 & 4728 & 14043 & -3 & $0.02 \%$ \\
\hline 500 & 14590 & 7376 & 21966 & 14579 & 7377 & 21956 & -10 & $0.05 \%$ \\
\hline 600 & 21021 & 10605 & 31626 & 21017 & 10614 & 31631 & 5 & $0.02 \%$ \\
\hline 700 & 28592 & 14458 & 43050 & 28629 & 14437 & 43066 & 16 & $0.04 \%$ \\
\hline 800 & 37434 & 18831 & 56265 & 37414 & 18848 & 56262 & -3 & $0.01 \%$ \\
\hline 900 & 47393 & 23839 & 71232 & 47374 & 23845 & 71219 & -13 & $0.02 \%$ \\
\hline 1000 & 58480 & 29453 & 87933 & 58508 & 29430 & 87938 & 5 & $0.01 \%$ \\
\hline
\end{tabular}


Table 5.2. Cumulative number of non-trivial primitive solutions of $x^{2}+2 y^{2}+2 z^{2}=t^{2}$

\begin{tabular}{|c|c|c|c|c|c|c|c|c|}
\hline limit $x$ & $\begin{array}{c}\text { exact } \\
\text { odd } \\
\text { count }\end{array}$ & $\begin{array}{c}\text { exact } \\
2 \times \text { odd } \\
\text { count }\end{array}$ & $\begin{array}{c}\text { exact } \\
\text { count }\end{array}$ & $\begin{array}{c}\text { asymptotic } \\
\text { odd count }\end{array}$ & $\begin{array}{c}\text { asymptotic } \\
2 \text { x odd } \\
\text { count }\end{array}$ & $\begin{array}{c}\text { asymptotic } \\
\text { count }\end{array}$ & $\begin{array}{c}\text { signed } \\
\text { difference }\end{array}$ & $\begin{array}{c}\text { percentage } \\
\text { relative } \\
\text { error }\end{array}$ \\
\hline 100 & 340 & 169 & 509 & 338 & 171 & 509 & 0 & $0.00 \%$ \\
\hline 200 & 1352 & 687 & 2039 & 1358 & 682 & 2040 & 1 & $0.05 \%$ \\
\hline 300 & 3058 & 1537 & 4595 & 3061 & 1535 & 4596 & 1 & $0.02 \%$ \\
\hline 400 & 5457 & 2716 & 8173 & 5446 & 2729 & 8175 & 2 & $0.02 \%$ \\
\hline 500 & 8509 & 4278 & 12787 & 8513 & 4265 & 12778 & -9 & $0.07 \%$ \\
\hline 600 & 12261 & 6137 & 18398 & 12262 & 6141 & 18403 & 5 & $0.03 \%$ \\
\hline 700 & 16702 & 8352 & 25054 & 16694 & 8359 & 25053 & -1 & $0.00 \%$ \\
\hline 800 & 21786 & 10941 & 32727 & 21809 & 10917 & 32726 & -1 & $0.00 \%$ \\
\hline 900 & 27602 & 13818 & 41420 & 27605 & 13817 & 41422 & 2 & $0.00 \%$ \\
\hline 1000 & 34096 & 17050 & 51146 & 34084 & 17057 & 51141 & -5 & $0.01 \%$ \\
\hline
\end{tabular}




\section{References}

[1] D. H. Bailey, J. M. Borwein, A. Mattingly and G. Wightwick, The computation of previously inaccessible digits of $\pi^{2}$ and Catalan's constant, Notices AMS 60(7) (2013), 844-854.

[2] E. T. Bell, The numbers of representations of integers in certain forms $a x^{2}+b y^{2}+c z^{2}$, Amer. Math. Monthly 31(3) (1924), 126-131.

[3] J. Choi and D. Cvijovic, Values of the polygamma functions at rational arguments, J. Phys. A: Math. Theor. 40 (2007), 15019-15028.

[4] J. Choi and D. Cvijovic, Corrigendum, Values of the polygamma functions at rational arguments, J. Phys. A: Math. Theor. 43 (2010), 239801.

[5] S. Cooper and M. D. Hirschhorn, On the number of primitive representations of integers as sums of squares, Ramanujan Journal 13 (2007), 7-25.

[6] S. Cooper and H. Y. Lam, On the Diophantine equation $n^{2}=x^{2}+b y^{2}+c z^{2}$, Journal of Number Theory 133 (2013), 719-737.

[7] L. E. Dickson, History of the Theory of Numbers, Vol. II, Carnegie Institute of Washington, Washington, Reprint: Chelsea, 1966, New York, 1920.

[8] L. E. Dickson, Introduction to the Theory of Numbers, University of Chicago Press, Reprint (1957), Dover Publications, 1929.

[9] E. Grosswald, Representations of Integers as Sums of Squares, Springer, New York, 1985.

[10] X. Guo, Y. Peng and H. Qin, On the representation numbers of ternary quadratic forms and modular forms of weight 3/2, Journal of Number Theory 140 (2014), $235-266$.

[11] W. Hürlimann, Exact and asymptotic evaluation of the number of distinct primitive cuboids, Journal of Integer Sequences 18(2), Article 15.2.5. (2015a).

[12] W. Hürlimann, On the number of primitive Pythagorean quintuples, Journal of Algebra, Number Theory: Advances and Applications 13(1) (2015b), 13-28.

[13] W. Hürlimann, Bell's ternary quadratic forms and Tunnel's congruent number criterion revisited, Advances in Pure Mathematics 5(5) (2015c), 267-277.

[14] W. Hürlimann, Cooper and Lam's conjecture for generalized Bell ternary quadratic forms, Journal of Number Theory 158 (2016a), 23-32.

[15] W. Hürlimann, On the number of primitive Pythagorean sextuples, Submitted for publication (2016b).

[16] A. Hurwitz, Somme de trois carrés, L'Intermédiaire des Mathématiciens 14 (1907), 106-107. (Mathematische Werke (1933), Vol. 2, 751).

[17] J. Kaczorowski, On a generalization of the Euler totient function, Monatsh. Math. 170 (2013), 27-48. 
[18] J. Kaczorowski and K. Wiertelak, On the sum of the twisted Euler function, Int. J. Number Theory 8(7) (2013), 1741-1761.

[19] J. Kaczorowski and K. Wiertelak, Omega theorems related to the general Euler totient function, J. Math. Anal. Appl. 412 (2014), 401-415.

[20] K. Kim and B.-K. Oh, The number of representations of squares by integral ternary quadratic forms, (2015).

URL: http://arxiv.org/abs/1509.09111.

[21] D. N. Lehmer, Asymptotic evaluation of certain totient sums, Amer. J. Math. 22(4) (1900), 293-335.

[22] Z. H. Sun and K. S. Williams, On the number of representations of $n$ by $a x^{2}+b x y+c y^{2}$, Acta Arithmetica 122(2) (2006), 101-171.

[23] Eric W. Weisstein, Dirichlet L-Series, From MathWorld - A Wolfram Web Resource, (1999).

$$
\text { http://mathworld.wolfram.com/DirichletL-Series.html. }
$$

[24] D. Ye, Representation of squares by certain ternary quadratic forms, Integers 14 (2014), \#A52. 